\title{
QT Interval Variability and QT-HP Coupling Strength in Amyotrophic Lateral Sclerosis Patients
}

\author{
Beatrice De Maria ${ }^{1}$, Gabriele Mora ${ }^{1}$, Kalliopi Marinou ${ }^{1}$, Riccardo Sideri ${ }^{1}$, Vlasta Bari ${ }^{2}$, \\ Beatrice Cairo $^{3}$, Emanuele Vaini ${ }^{2}$, Laura Adelaide Dalla Vecchia*1 and Alberto Porta*2,3 \\ ${ }^{1}$ IRCCS Istituti Clinici Scientifici Maugeri, Milan, Italy \\ ${ }^{2}$ Department of Cardiothoracic, Vascular Anesthesia and Intensive Care, IRCCS Policlinico San \\ Donato, San Donato Milanese, Milan, Italy \\ ${ }^{3}$ Department of Biomedical Sciences for Health, University of Milan, Milan, Italy
}

\begin{abstract}
In the recent years we have witnessed an increasing interest in studying cardiac control in amyotrophic lateral sclerosis (ALS) patients. The variability of the overall duration of cardiac electrical activity comprising depolarization and repolarization periods, namely the $Q T$ interval, could provide information about the cardiac control of ALS patients complementary to that derived from the variability of heart period (HP). In this study we evaluate first the HP and $Q T$ variabilities in $10 \mathrm{ALS}$ patients at rest in supine position (REST) and during $75^{\circ}$ head-up tilt (TILT). The QT interval was approximated as RTapex and RTend intervals, representing the temporal distance between $R$-wave peak and $T$-wave apex and end, respectively. HP was taken as the time distance between two consecutive $R$-wave peaks. Time and frequency domain markers were computed over HP, RTa and RTe beat-to-beat series. The RTa-HP and RTe-HP squared coherence was calculated as well. We found that time and frequency domain indexes derived from $Q T$ variability changed during TILT in the direction expected for a healthy population. Frequency domain HP variability markers showed a blunted response to TILT. RTa-HP and $R T$-HP squared coherence did not vary during TILT. QT variability and QT-HP coupling markers in ALS patients showed an apparently normal response to TILT not fully mirrored by HP variability indexes.
\end{abstract}

\section{Introduction}

Amyotrophic lateral sclerosis (ALS) is a neurodegenerative disease characterized by the loss of the upper and lower motoneurons. In the last years, signs and symptoms of autonomic nervous system involvement have been described in ALS, including subclinical dysfunction of cardiovascular, sudomotor, gastrointestinal, salivary and lacrimal regulations. At the cardiovascular control level, impaired baroreflex response and blunted response to postural stressor have been described [1-3]. Nevertheless, results about the autonomic nervous system involvement in ALS are still controversial. In addition, no clear relation between autonomic disturbances and patients' clinical characteristics and prognosis have been identified. New approaches to the study of the cardiac control of ALS patients are needed to improve the characterization of ALS patients. The study of the variability of the time taken for ventricular depolarization and repolarization, approximated by the QT interval measured on the electrocardiogram (ECG), could provide new insight. This analysis has been demonstrated to be useful in describing the sympathetic drive directed to the heart, in particular to the ventricles, with prognostic meaning [4,5]. To the best of our knowledge, only two studies quantified the QT interval in ALS patients [6,7], but none of them monitored the QT variability and the coupling strength between QT and heart period (HP) variations.

In this study we test the hypothesis that the analysis of the QT variability can be performed in ALS patients and we evaluate the changes of QT variability during a postural challenge in ALS patients. Indexes of QT variability were examined with HP variability markers to assess potential additional information. QT-HP coupling strength was monitored as well.

\section{Experimental protocol and analysis}

\subsection{Experimental protocol}

We enrolled 10 ALS patients (2 males; age: $62.5 \pm 7.55$ years; body mass index: $22.76 \pm 4.08 \mathrm{~kg} \cdot \mathrm{m}^{-2}$ ). Eight patients had spinal onset, while 2 patients had bulbar onset. The time from diagnosis was $27.9 \pm 16.38$ months. The study was conducted at IRCCS Istituti Clinici Scientifici Maugeri, Milan, Italy, with the approval of the Local 

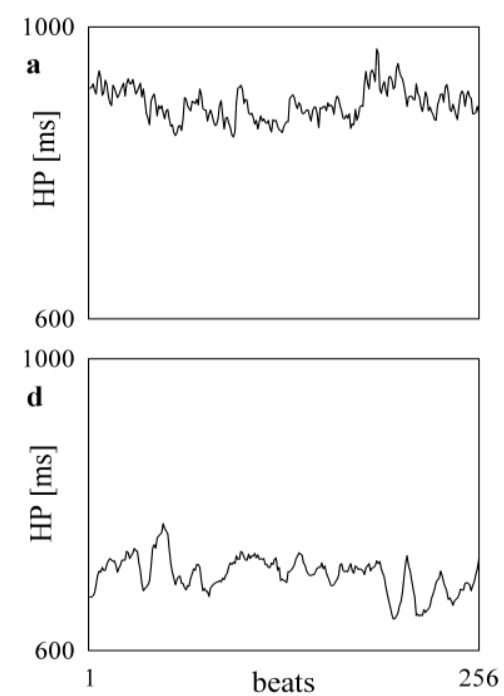
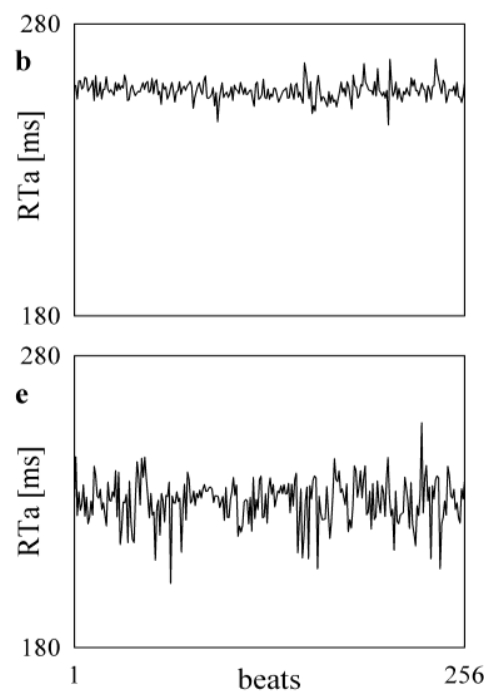
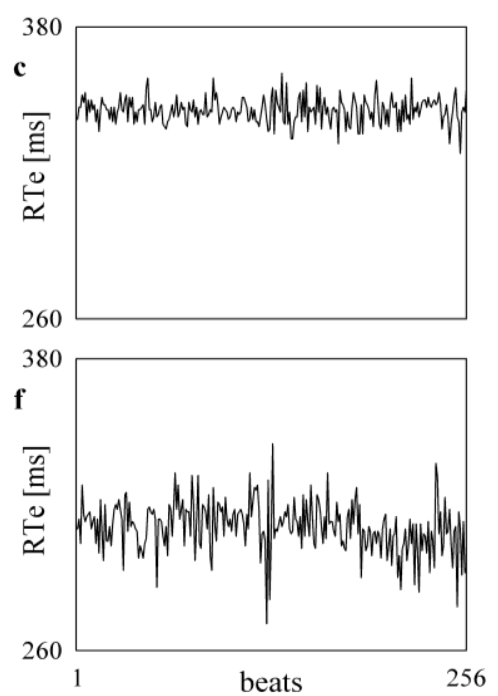

Figure 1. Examples of HP (a,d), RTa (b,e) and RTe (c,d) variability series at REST (a,b,c) and during TILT (d,e,f).

Ethics Committee (CE1077). The study is in keeping with the principles of the Declaration of Helsinki and each subject signed a written informed consent.

All the subjects were instructed to avoid caffeinated beverages in the 24 hours preceding the test and reached the laboratory by wheelchair. During the experiment ECG (modified lead II) was acquired at $1000 \mathrm{~Hz}$. Thoracic movements were monitored as well to estimate breathing rate. The signals were recorded for 10 minutes at rest in supine position (REST) and for 5 minutes during head-up tilt test at $75^{\circ}$ (TILT). All the patients completed the experiments without signs of pre-syncope.

\subsection{Beat-to-beat time series extraction}

The HP was approximated as the temporal distance between two consecutive R-wave peaks detected over the ECG. QT interval was approximated as the temporal distance between the $\mathrm{R}$-wave peak and the $\mathrm{T}$-wave apex (RTa) and as the temporal distance between the R-wave peak and T-wave end (RTe) [8]. Both the apexes of Rwave and T-wave were fixed using parabolic interpolation [8]. The end of the T-wave was identified as the time when the first derivative, calculated on the $\mathrm{T}$ wave downslope, became smaller of the $30 \%$ of the maximum first derivative value [8]. The $i$ th RTa and RTe measures always followed the $i$ th HP, thus linking the ith RTa and RTe to the previous HP. The detections of the R-wave peaks, T-wave apexes and $\mathrm{T}$-wave ends were visually checked and manually corrected in case of erroneous identifications. In case of ectopic beats, the series were corrected by means of linear interpolation. Corrections never exceeded 5\% of the total values analyzed in each session. HP, RTa and RTe series of 256 consecutive values were selected randomly in each experimental session (i.e. REST or TILT). Examples of HP, RTa and RTe series at REST and during TILT are shown in Fig.1. We computed mean and variance of all series indicated, respectively, as $\mu_{\mathrm{HP}}, \mu_{\mathrm{RT}}, \mu_{\mathrm{RTe}}$ and $\sigma_{\mathrm{HP}}^{2}$, $\sigma^{2}{ }_{\mathrm{RTa}}, \sigma^{2}{ }_{\mathrm{RTe}}$. Means were expressed in $\mathrm{ms}$, while variances in $\mathrm{ms}^{2}$.

\subsection{Power spectral analysis}

Parametric power spectral analysis was performed on HP, RTa and RTe series. The series were described by an autoregressive model, whose order was chosen according to Akaike information criterion. Power spectral density was estimated from the identified coefficient of the autoregressive model and the variance of the prediction error [9]. The power spectral density was decomposed into spectral components. Each component was classified as low frequency (LF, 0.04-0.15 Hz) or high frequency (HF, $0.15-0.5 \mathrm{~Hz}$ ) according to their central frequency [9]. The sum of the LF components of HP, RTa and RTe was expressed in absolute units (i.e. $\mathrm{ms}^{2}$ ) and termed, respectively, as $\mathrm{LF}_{\mathrm{a}, \mathrm{HP}}, \mathrm{LF}_{\mathrm{a}, \mathrm{RTa}}$ and $\mathrm{LF}_{\mathrm{a}, \mathrm{RTe}}$. The $\mathrm{LF}_{\mathrm{a}, \mathrm{HP}}$

Table 1. Time domain HP and QT variability indexes

\begin{tabular}{lcc}
\hline \multicolumn{1}{c}{ Index } & REST & TILT \\
\hline$\mu_{\mathrm{HP}}[\mathrm{ms}]$ & $871.19 \pm 138.73$ & $776.41 \pm 82.57^{*}$ \\
$\mu_{\mathrm{RTa}}[\mathrm{ms}]$ & $269.48 \pm 20.13$ & $260.26 \pm 19.32^{*}$ \\
$\mu_{\text {RTe }}[\mathrm{ms}]$ & $342.17 \pm 19.66$ & $328.89 \pm 10.9^{*}$ \\
$\sigma_{\text {HP }}^{2}\left[\mathrm{~ms}^{2}\right]$ & $842.37 \pm 1058.57$ & $531.5 \pm 536.84$ \\
$\sigma_{\text {RTa }}^{2}\left[\mathrm{~ms}^{2}\right]$ & $19.08 \pm 21.52$ & $59.38 \pm 73.75^{*}$ \\
$\sigma_{\text {RTe }}^{2}\left[\mathrm{~ms}^{2}\right]$ & $84.66 \pm 73.64$ & $165.81 \pm 159.23^{*}$ \\
\hline
\end{tabular}

REST: at rest in supine position; TILT: head-up tilt; HP: heart period; RTa: R-wave peak to T-wave apex; RTe: Rwave peak to T-wave end; $\mu_{\mathrm{HP}}$ : HP mean; $\mu_{\mathrm{RT}}$ : RTa mean; $\mu_{\mathrm{RTe}}$ : RTe mean; $\sigma_{\mathrm{HP}}^{2}$ : HP variance; $\sigma^{2}{ }_{\mathrm{RTa}}: \mathrm{RTa}$ variance; $\sigma^{2}{ }_{\text {RTe }}:$ RTe variance. The symbol $*$ indicates $p<0.05$ versus REST. 
Table 2. HP and QT power spectral markers

\begin{tabular}{lcc}
\hline \multicolumn{1}{c}{ Index } & REST & TILT \\
\hline $\mathrm{LF}_{\mathrm{a}, \mathrm{HP}}\left[\mathrm{ms}^{2}\right]$ & $125.72 \pm 181.21$ & $121.4 \pm 152.69$ \\
$\mathrm{LF}_{\mathrm{nu}, \mathrm{HP}}[\mathrm{nu}]$ & $46.47 \pm 18.83$ & $52.95 \pm 26.08^{*}$ \\
$\mathrm{HF}_{\mathrm{a}, \mathrm{HP}}\left[\mathrm{ms}{ }^{2}\right]$ & $91.32 \pm 95.65$ & $66.64 \pm 64.4$ \\
$\mathrm{HF}_{\mathrm{nu}, \mathrm{HP}}[\mathrm{nu}]$ & $48.51 \pm 17.32$ & $39.51 \pm 23.07$ \\
$\mathrm{LF}_{\mathrm{a}, \mathrm{RTa}}\left[\mathrm{ms}^{2}\right]$ & $4.81 \pm 5.32$ & $14.93 \pm 17.18^{*}$ \\
$\mathrm{LF}_{\mathrm{a}, \mathrm{RTe}}\left[\mathrm{ms}^{2}\right]$ & $20.59 \pm 16.76$ & $54.65 \pm 69.05^{*}$ \\
\hline
\end{tabular}

REST: at rest in supine position; TILT: head-up tilt; HP: heart period; RTa: R-wave peak to T-wave apex; RTe: Rwave peak to T-wave end; LF: low frequency; HF: high frequency; $\mathrm{LF}_{\mathrm{a}, \mathrm{HP}}$, absolute power of HP series in LF band; $\mathrm{LF}_{\mathrm{nu}, \mathrm{HP}}$ : normalized power of HP series in $\mathrm{LF}$ band; $\mathrm{HF}_{\mathrm{a}, \mathrm{HP}}$ : absolute power of $\mathrm{HP}$ series in $\mathrm{HF}$ band; $\mathrm{HF}_{\mathrm{nu}, \mathrm{HP}}$ : normalized power of $\mathrm{HP}$ series in $\mathrm{HF}$ band; $\mathrm{LF}_{\mathrm{a}, \mathrm{RTa}}$, absolute power in $\mathrm{LF}$ band of $\mathrm{RTa}$ variability; $\mathrm{LF}_{\mathrm{a}, \mathrm{RTe}}$ : absolute power of RTe series in LF band. The symbol * indicates $p<0.05$ versus REST.

power can be considered to be a marker of the sympathetic and vagal modulation directed to the sinus node [9], while $\mathrm{LF}_{\mathrm{a}, \mathrm{RTa}}$ and $\mathrm{LF}_{\mathrm{a}, \mathrm{RTe}}$ powers to be markers of sympathetic modulation directed to the ventricles [4,5]. The sum of the HF components of HP was expressed in absolute units (i.e. $\mathrm{ms}^{2}$ ) and labelled as $\mathrm{HF}_{\mathrm{a}, \mathrm{HP}}$. The $\mathrm{HF}_{\mathrm{a}, \mathrm{HP}}$ power is a marker of the vagal modulation directed to the sinus node [9]. For HP series, LF and HF powers were also expressed in normalized units ( $\mathrm{LF}_{\mathrm{nu}, \mathrm{HP}}$ and $\mathrm{HF}_{\mathrm{nu}, \mathrm{HP}}$, respectively) as the ratio of $\mathrm{LF}_{\mathrm{a}, \mathrm{HP}}$ and $\mathrm{HF}_{\mathrm{a}, \mathrm{HP}}$ to $\sigma_{\mathrm{HP}}^{2}$ minus the power of components with central frequencies below $0.04 \mathrm{~Hz}$ [10]. Normalization makes the $\mathrm{LF}_{\mathrm{nu}, \mathrm{HP}}$ index more linked to sympathetic modulation directed to sinus node than the $\mathrm{LF}_{\mathrm{a}, \mathrm{HP}}$ power [10].

\subsection{Squared coherence analysis}

The assessment of the coupling strength between HP and RTa, or RTe, was performed by exploiting the squared coherence function $\mathrm{K}^{2}$. It represents the degree of linear correlation between the two series as a function of the frequency. $\mathrm{K}^{2}$ was calculated as the ratio between the square cross-spectrum modulus between HP and RTa, or $\mathrm{RTe}$, to the product of the power spectra of RTa, or RTe, and HP series. The estimation of the cross-spectrum was grounded over the identification of a bivariate autoregressive process and the model order was set to 10 [5]. $\mathrm{K}^{2}$ was sampled in correspondence of the weighted central frequency of the HP components in LF and HF bands, where the weights were the powers of the components [5]. Markers in the LF and HF bands were

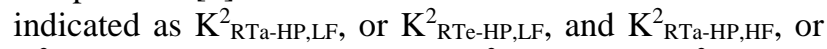
$\mathrm{K}^{2}{ }_{\mathrm{RTe}}$-HP,HF, $\quad$ respectively. $\quad \mathrm{K}^{2}{ }_{\mathrm{RTa} \text {-HP,LF, }} \mathrm{K}^{2}{ }_{\mathrm{RTe} \text {-HP,LF }}$, $\mathrm{K}^{2}{ }_{\mathrm{RTa}} \mathrm{HP}, \mathrm{HF}$ and $\mathrm{K}^{2} \mathrm{RTa}$-HP,HF ranged from 1 (full correlation) to 0 (null correlation) and were dimensionless.
Table 3. QT-HP coupling strength parameters

\begin{tabular}{ccc}
\hline Index & REST & TILT \\
\hline $\mathrm{K}^{2}$ RTa-HP,LF & $0.24 \pm 0.18$ & $0.17 \pm 0.12$ \\
$\mathrm{~K}^{2}{ }_{\text {RTa-HP,HF }}$ & $0.19 \pm 0.26$ & $0.13 \pm 0.12$ \\
$\mathrm{~K}^{2}{ }_{\text {RTe-HP,LF }}$ & $0.14 \pm 0.1$ & $0.14 \pm 0.15$ \\
$\mathrm{~K}^{2}$ RTe-HP,HF & $0.1 \pm 0.07$ & $0.12 \pm 0.08$ \\
\hline
\end{tabular}

REST: at rest in supine position; TILT: head-up tilt; HP: heart period; RTa: R-wave peak to T-wave apex; RTe: Rwave peak to T-wave end; $\mathrm{K}^{2}$ : squared coherence; LF: low frequency; HF: high frequency; $\mathrm{K}^{2}{ }_{\mathrm{RTa}-\mathrm{HP}, \mathrm{LF}}: \mathrm{K}^{2}$ between $\mathrm{RTa}$ and $\mathrm{HP}$ series in the LF band; $\mathrm{K}^{2}{ }_{\mathrm{RT} \text { Ta HP,HF}}: \mathrm{K}^{2}$ between $\mathrm{RTa}$ and HP series in the HF band; $\mathrm{K}^{2}$ RTe-HP,LF: $\mathrm{K}^{2}$ between RTe and HP series in the LF band; $\mathrm{K}^{2}{ }_{\mathrm{RT} \text { Te-HP,HF: }} \mathrm{K}^{2}$ between $\mathrm{RTe}$ and HP series in the HF band.

\subsection{Statistical analysis}

The significance of the differences of HP variability, QT variability and $\mathrm{K}^{2}$ indexes between the two experimental conditions (i.e. REST and TILT) was tested via paired ttest, or Wilcoxon signed rank test when appropriate. Data were reported as mean \pm standard deviation. A $p<0.05$ was always considered as significant.

\section{Results}

Table 1 shows the results of the analysis of HP, RTa and $\mathrm{RTe}$ variability in the time domain. $\mu_{\mathrm{HP}}, \mu_{\mathrm{RTa}}$ and $\mu_{\mathrm{RT}}$ decreased during TILT compared to REST. $\sigma^{2}$ RTa and $\sigma^{2}{ }_{\text {RTe }}$ significantly increased during TILT compared to REST, while $\sigma^{2}$ HP did not change. Table 2 shows the results of the power spectral analysis of HP, RTa and RTe variability. $L_{a, R T a}$ and $L_{a, R T e}$ powers significantly increased during TILT in ALS patients. Only the $\mathrm{LF}_{\mathrm{nu}, \mathrm{HP}}$ increased during TILT, while none of the other HP variability spectral markers changed significantly. Table 3 shows the results of the $\mathrm{K}^{2}$ analysis between HP and RTa, or RTe, in both LF and HF bands. Both RTa-HP and RTe-HP coupling strength remained stable across the experimental condition and this result held regardless of the frequency band.

\section{Discussion}

The main novelty of this study is the assessment of QT variability at REST and during TILT in ALS patients, together with the more traditional evaluation of HP variability. We found that QT variability can be analyzed in ALS patients and might represent a complementary tool with respect to the HP variability to investigate the cardiac control in ALS. Indeed, results showed that $\mu_{\mathrm{RTa}}$ and $\mu_{\mathrm{RT}}$ decreased during TILT, as the consequence of the shortening of the HP and the QT-RR relationship. As observed in healthy subjects [5,11], the variance of QT, approximated by $\sigma^{2}{ }_{\mathrm{RTa}}$ and $\sigma^{2}$ RTe, and the QT variability 
power in $\mathrm{LF}$ band, approximated by $\mathrm{LF}_{\mathrm{a}, \mathrm{RTa}}$ and $\mathrm{LF}_{\mathrm{a}, \mathrm{RTe}}$, increased during TILT as a consequence of the sympathetic activation induced by the orthostatic challenge. We conclude that the typical response of QT variability to TILT is preserved in our group of ALS patients. This result is in agreement with [7] who did not observed abnormalities in the QT interval of ALS patients. Remarkably, ALS patients were characterized by a stable degree of correlation between HP and QT variability during TILT. This result is at difference with healthy subjects $[5,12]$. Given the low level of QT-HP coupling observed in this study even at REST, this finding might indicate that the QT-HP relation is impaired in ALS patients and this impairment might favor the QT dispersion. It is worth noting that an increase of QT dispersion was observed in the terminal stages of ALS compared to initial stages and this increase was taken as a hallmark of an increased risk of sudden death [6].

As to the HP variability, ALS individuals showed a limited response to TILT compared to QT variability. Indeed, while the decrease of $\mu_{\mathrm{HP}}$ and the increase of $\mathrm{LF}_{\mathrm{nu}, \mathrm{HP}}$ power in reaction to TILT was found, a significant decrease of $\sigma^{2}{ }_{\mathrm{HP}}$ and $\mathrm{HF}_{\mathrm{a}, \mathrm{HP}}$ power was not observed. This peculiar response of HP variability to TILT was already documented in [1,2]. This blunted response of HP variability to TILT in connection with the preserved QT variability and QT-HP coupling strength suggest that the analysis of QT variability might provide information complementary to that of HP variability.

\section{Conclusion}

In this study, we evaluated first the QT variability in ALS patients during an orthostatic challenge. We found that the sympathetic modulation directed to the heart, as quantified by QT variability analysis, increased in response to the postural change. However, modifications of the HP variability parameters were blunted and the expected reduction of the degree of association between HP and QT with TILT was absent. However, even though the response of QT variability to postural maneuver is preserved [5,11], the lack of comparison with age-matched control group does not allow us to fully understand the magnitude of this response. Moreover, the preservation of QT-HP coupling with TILT stresses the need of studying the QT-HP relationship via, for example, ad-hoc modeling approaches [11,12].

In general, the comparison between findings derived from QT and HP variability series allows us to conclude that the QT variability in ALS patients might provide information complementary to that given by the exclusive analysis of HP variability and might offer a more precise picture about the cardiac control of ALS patients.

\section{Acknowledgments}

The study was partially supported by AriSLA, Fondazione Italiana di Ricerca per la Sclerosi Laterale Amiotrofica.

\section{References}

[1] L. Dalla Vecchia et al., "Cardiovascular neural regulation is impaired in amyotrophic lateral sclerosis patients. A study by spectral and complexity analysis of cardiovascular oscillations," Physiol. Meas., vol. 36, pp. 659-670, 2015.

[2] B. De Maria et al., "Cardiovascular control indexes in amyotrophic lateral sclerosis patients and their relation with clinical markers," Conf. Proc. IEEE Eng. Med. Biol. Soc., vol. 2015, pp. 2055-2058, 2015.

[3] B. Cairo et al., "Information-domain method for the quantification of the complexity of the sympathetic baroreflex regulation in healthy subjects and amyotrophic lateral sclerosis patients," Physiol. Meas., vol. 40, art. no. 034004, 2019.

[4] A. Porta et al., "Autonomic control of heart rate and QT interval variability influences arrhythmic risk in long QT syndrome type 1," J. Am. Coll. Cardiol., vol. 65, pp. 367 374, 2015.

[5] A. Porta et al., "Frequency domain assessment of the coupling strength between ventricular repolarization duration and heart period during graded head-up tilt," J. Electrocardiol., vol. 44, pp. 662-668, 2011.

[6] H. Asai et al., "Sympathetic disturbances increase risk of sudden cardiac arrest in sporadic ALS," J. Neurol. Sci., vol. 254, pp. 78-83, 2007.

[7] H. C. Timmins et al., "Cardiometabolic health and risk of amyotrophic lateral sclerosis," Muscle Nerve, vol. 56, pp. 721-725, 2017.

[8] A. Porta et al., "Performance assessment of standard algorithms for dynamic R-T interval measurement: comparison between R-Tapex and R-Tend approach," Med. Biol. Eng. Comput., vol. 36, pp. 35-42, 1998.

[9] Task Force of the European Society of Cardiology and the North American Society of Pacing and Electrophysiology, "Heart rate variability. Standards of measurement, physiological interpretation, and clinical use." Eur. Heart J., vol. 17, pp. 354-381, 1996.

[10] M. Pagani et al., "Power spectral analysis of heart rate and arterial pressure variabilities as a marker of sympatho-vagal interaction in man and conscious dog," Circ. Res., vol. 59, pp. 178-193, 1986.

[11] A. Porta, E. Tobaldini, T. Gnecchi-Ruscone and N. Montano, "RT variability unrelated to heart period and respiration progressively increases during graded head-up tilt," Am. J. Physiol., vol. 298, pp. H1406-H1414, 2010.

[12] A Porta, V. Bari, B. De Maria and M. Baumert, "A network physiology approach to the assessment of the link between sinoatrial and ventricular cardiac controls," Physiol. Meas., vol. 38, pp. 1472-1489, 2017.

Address for correspondence:

Beatrice De Maria,

IRCCS Istituti Clinici Scientifici Maugeri,

Via Camaldoli 64, 20138 Milano, Italy,

Tel: +390250725286

email: beatrice.demaria@icsmaugeri.it 